\title{
A Thirty-Five-Minute Nap Improves Performance and Attention in the 5-m Shuttle Run Test during and outside Ramadan Observance
}

\author{
Hsen Hsouna ${ }^{1,2}$, Omar Boukhris ${ }^{1,2} \mathbb{D}$, Khaled Trabelsi 2,3 $\mathbb{D}$, Raouf Abdessalem 1,2, \\ Achraf Ammar ${ }^{4}\left(\mathbb{D}\right.$, Jordan M. Glenn ${ }^{5,6}{ }^{(D}$, Nick Bott ${ }^{6,7}$, Nizar Souissi ${ }^{1,2}$, Paola Lanteri ${ }^{8}(\mathbb{D}$, \\ Sergio Garbarino 9 (D), Nicola Luigi Bragazzi $9,10,11, *,+(\mathbb{D})$ and Hamdi Chtourou $1,2,+(\mathbb{D}$
}

1 "Physical Activity, Sport and Health" Research Unit, UR18JS01, National Sport Observatory, Tunis 1003, Tunisia; hsen.hsouna92@gmail.com (H.H.); omarboukhris24@yahoo.com (O.B.);

raoufabdesalem18@gmail.com (R.A.); n_souissi@yahoo.fr (N.S.); h_chtourou@yahoo.fr (H.C.)

2 High Institute of Sport and Physical Education of Sfax, University of Sfax, Sfax 3000, Tunisia; trabelsikhaled@gmail.com

3 Research Laboratory: Education, Motricity, Sport and Health, EM2S, LR19JS01, High Institute of Sport and Physical Education of Sfax, University of Sfax, Sfax 3000, Tunisia

4 Institute of Sport Science, Otto-von-Guericke University Magdeburg, Universitätsplatz 2, 39106 Magdeburg, Germany; Ammar.achraf@ymail.com

5 Department of Health, Exercise Science Research Center Human Performance and Recreation, University of Arkansas, Fayetteville, AR 72701, USA; jordan@neurotrack.com

6 Neurotrack Technologies, 399 Bradford St. Redwood City, CA 94063, USA; nick@neurotrack.com

7 Department of Medicine, Clinical Excellence Research Center, Stanford University School of Medicine, Stanford, CA 94305, USA

8 Neurophysiopathology Unit, Foundation IRCCS Carlo Besta Neurological Institute, 20133 Milan, Italy; paola.lanteri@istituto-besta.it

9 Department of Neuroscience, Rehabilitation, Ophthalmology, Genetics, Maternal and Child Health (DINOGMI), University of Genoa, 16132 Genoa, Italy; sgarbarino.neuro@gmail.com

10 Department of Health Sciences (DISSAL), Postgraduate School of Public Health, University of Genoa, 16132 Genoa, Italy

11 Department of Mathematics and Statistics, Laboratory for Industrial and Applied Mathematics (LIAM) York University, Toronto, ON M3J 1P3, Canada

* Correspondence: robertobragazzi@gmail.com

+ These authors contributed equally.

Received: 27 May 2020; Accepted: 6 July 2020; Published: 11 July 2020

\begin{abstract}
Ramadan observance is characterized by several changes in behaviors, such as food and sleep, which could affect physical and cognitive performance. The aim of the present study was to investigate the effects of a 35-min nap (N35) opportunity on physical performance during the 5-m shuttle run test (5mSRT); attention; feelings; mood states; and perceptual measures of stress, fatigue, and muscle soreness during Ramadan observance. Fourteen physically active men (22 \pm 3 years, $177 \pm 4 \mathrm{~cm}, 76 \pm 5 \mathrm{~kg}$ ) were tested after a no-nap condition (N0), N35 15 days before Ramadan (BR), the last 10 days of Ramadan (DR), and 20 days after Ramadan (AR). Measures included the digit cancellation test (attention estimation), the profile of mood state (POMS), and the Hooper questionnaires. After a 5-min standard warm-up, participants performed the $5 \mathrm{mSRT}(6 \times 30 \mathrm{~s}$ with $35 \mathrm{~s}$ in between; best distance (BD), total distance (TD), and fatigue index (FI) were recorded), along with the rating of perceived exertion (RPE) after each test repetition. After the 5mSRT test, participants responded to the feeling scale (FS). The results showed that TD and FI during the 5mSRT were not affected by Ramadan observance. However, BD was significantly lower than DR compared to AR after N0 $(\Delta=-4.3 \pm 1.3 \% ; p<0.01)$ and N35 $(\Delta=-2.6 \pm 1.0 \% ; p<0.05)$. After N0, attention decreased significantly at DR in comparison with BR $(p<0.05)$ and AR $(p<0.001)$. BD and TD improved after N35 compared to N0 at BR $(\Delta=+4.4 \pm 2.1 \%, p<0.05$ for BD and $\Delta=+4.8 \pm 1.6 \%, p<0.01$ for TD),
\end{abstract}


$\mathrm{DR}(\Delta=+7.1 \pm 2.2 \%, p<0.05$ for BD and $\Delta=+5.1 \pm 1.6 \%, p<0.01$ for TD $)$, and AR $(\Delta=+5.5 \pm 1.5 \%$, $p<0.01$ for $\mathrm{BD}$ and $\Delta=+5.2 \pm 1.2 \%, p<0.001$ for TD). A significant increase in attention was observed after N35 in comparison with N0 at DR $(p<0.01)$ and AR $(p<0.01)$. However, no changes were found for the perception of mood states, stress, sleep, muscle soreness, and the FI during the 5mSRT. Also, N35 was better than N0 for RPE at DR $(p<0.05)$, feelings at AR $(p<0.05)$, and fatigue estimation at AR $(p<0.01)$. A 35-min nap opportunity may have beneficial effects on physical and cognitive performances before, during, and after Ramadan.

Keywords: performance; exercise; fasting; psychological; siesta

\section{Introduction}

Intermittent fasting is an obligation for healthy pubescent Muslims during the month of Ramadan (observance lasting between 29 and 30 days) [1]. During this month, it is prohibited for Muslims to eat, drink, smoke, or have sexual intercourse from dawn to sunset (fasting period) [2]. As a result, these obligations reduce diurnal and increase nocturnal social activities in mainly Muslims countries [3].

Changes in eating schedules as well as other activities (i.e., late prayer (Taraweeh)) have considerable effects on sleep [4-7] and food/fluid intakes [8,9]. Other changes may also be observed during this month [10,11]. For example, Chtourou et al. [3] reported changes in mood states and participant chronotype during the month of Ramadan. With these changes, there is the possibility of negative effects on physical [12-15] and cognitive [11] performances during this month.

Boukhris et al. [11] reported a significant decrease in sleep efficiency and duration during and after Ramadan compared to before the fasting month. Similarly, perception of sleep quality was reported to be lower during compared to before Ramadan [16]. In this context, it has been reported that light-sleep stage duration increases significantly during Ramadan. This may be attributed to an increase in the number of awakenings, reflecting increased nighttime metabolism due to the late Souhour meal (last meal before starting the day fast) [17]; it may also be attributed to increased food-seeking behaviors. Additionally, Zerguini et al. [18] suggested that poor sleep quality during Ramadan could be explained by the accommodation of late evening food intake. This reduction in sleep duration may impede physical performance as studies report that sleep reduction has negative impacts on cognitive $[19,20]$ and physical $[21,22]$ performance. As adequate sleep (quantity and quality) is important for maximizing cognitive and physical performance as well as physical recovery [23], recent studies suggest that the inclusion of a short nap during the daytime (i.e., between $13 \mathrm{~h}$ and $15 \mathrm{~h}$ ) may help overcome these detrimental effects in performance. Previously, beneficial effects of a 30-min nap on 20-m sprint times have been reported [24]. When repeated sprint exercise was completed after a bout of sleep deprivation, Hammouda et al. [25] reported positive effects from 20-min and 90-min nap opportunities. The nap-related beneficial effects also exist after a night of normal sleep (25-min nap [26,27] and 45-min nap [28]). For example, during the 5-m shuttle run test (5mSRT), Boukhris et al. [26] and Abdessalem et al. [27] reported that a 25-min nap enhanced physical performance. In this way, Hammouda et al. [25] suggested that a nap opportunity could represent a full sleep cycle, which contains slow wave sleep (SWS) and rapid eye movement (REM) sleep stages. SWS is a known recovery period for daily metabolism. Thus, Boukhris et al. [26] reported that the beneficial effect of nap on physical performance could be explained by an improvement of alertness and reduction of sleepiness and fatigue. As the effects of Ramadan on sleep may represent a series of sleep abnormalities, the inclusion of a nap after sleep during this period may be beneficial for physical and cognitive performance.

Therefore, the aim of the present study was to investigate the effects of a 35-min nap before, during, and after Ramadan on physical and cognitive performance in physically active men. In a recent study, Hsouna et al. [29] showed that 25-min of nap opportunity had no significant effect on 
the $5 \mathrm{mSRT}$ performance and attention before and during Ramadan; a significant positive effect was reported only after Ramadan. The authors suggested that longer nap duration is required to elicit a significant effect on performance during Ramadan. Previous studies evaluating the effects of different nap durations (i.e., $25 \mathrm{~min}, 35 \mathrm{~min}$, and $45 \mathrm{~min}$ ) on physical and cognitive performance showed a more beneficial effect of nap of $35 \mathrm{~min}$ and $45 \mathrm{~min}$ durations compared to $25 \mathrm{~min}[26,28]$. Therefore, we hypothesized that 35-min of nap opportunity before and during Ramadan would improve performance in both categories. We also collected mood states, subjective perception of stress, muscle soreness, fatigue, and sleep quality. We hypothesized that the 35-min nap opportunity would overcome the previously reported negative impacts of Ramadan on mood states [3] and sleep quality [16]. Finally, as Boukhris et al. [11] reported that the reductions in sleep duration during Ramadan were still present after the fasting month, we hypothesized that a 35-min nap would also be beneficial after Ramadan.

\section{Materials and Methods}

\subsection{Participants}

A minimum of eleven participants was required for inclusion in the present study. This sample size was calculated using the software $G^{*}$ power (version 3.1.9.2; Kiel University, Kiel, Germany, $\alpha=0.05$ and power $=0.95)$ and based on the effect size $(=0.46)$ shown by Herrera et al. [30]. Due to potential dropout, twenty participants were recruited. Five participants were excluded from the data analysis as they did not complete all required sessions $(n=3)$ or failed to initiate sleep $(n=2)$. After introducing the study and obtaining their written consent, fourteen physically active males (age: $22 \pm 3$ years, height: $177 \pm 4 \mathrm{~cm}$, body-mass: $76 \pm 5 \mathrm{~kg}$ ) participated in the present study. The participants were recruited by advertising in classes and posting notices on bulletin boards. They did not have any pathological disorders, and they regularly performed fast walking and jogging at $3 \times 1$-h sessions/week in the late afternoon during the study. The study was conducted according to the Declaration of Helsinki and was fully approved by the local ethics committee (CPP: 0098/2018).

\subsection{Experimental Design}

All participants were familiarized to the protocol and tests of the present study. They then participated randomly in two testing sessions: a no-nap opportunity (N0) and a 35-min nap opportunity (N35). Each testing session occurred during three separate occasions: 15 days before Ramadan (BR), the last 10 days of Ramadan (DR), and 20 days after Ramadan (AR). At least $72 \mathrm{~h}$ was required between successive testing sessions. During each condition, participants came to the laboratory for a period of time between $13 \mathrm{~h} 45$ and $18 \mathrm{~h} 30$. For the nap, participants were allotted $15 \mathrm{~min}$ to be accustomed to the new place of sleep in the sleep laboratory. Following the $15 \mathrm{~min}$ acclimation period, participants completed the 35-min nap in dark and quiet sleep rooms. After N35 and during N0, participants did not engage in physical activity but were allowed to perform leisure activities such as watching $\mathrm{TV}$, playing video games, etc. in a comfortable armchair. At 17h00, participants completed the digit cancellation test (assessment of attention), 5-min of standardized warm-up, and the 5mSRT $(6 \times 30 \mathrm{~s}$ with $35 \mathrm{~s}$ in between). The digit cancellation test consists of deleting target numbers (i.e., numbers composed by three grouped digits) on a sheet of randomly arranged possibilities; the sum of correct responses was recorded for analysis.

During BR, DR, and AR (after the nap conditions), after the end of the 5mSRT, the Hooper questionnaire was used to estimate sleep, stress, fatigue, and muscle soreness using subjective scales ranging from "1" (very low or good) to "7" (very high or bad) [31]. During the same period, before the $5 \mathrm{mSRT}$ at $16 \mathrm{~h} 45$, the profile of mood states (POMS) questionnaire was used to evaluate seven states (i.e., tension, depression, anger, vigor, fatigue, confusion, and interpersonal relationships), where participants respond to 65 adjectives on a scale ranging from " 0 " (not at all) to " 4 " (extreme) [32].

Rating of perceived exertion (RPE) was estimated after each repetition of the $5 \mathrm{mSRT}$ on a scale ranging from " 0 " (very light) to " 10 " (very hard) [33]. The mean score during the $5 \mathrm{mSRT}$ was calculated 
for analysis. Feelings (FS) scores were recorded after the $5 \mathrm{mSRT}$ on a scale ranging from " -5 " (feeling very displeased) to " +5 " (feeling pleasure) by responding to the question: "How are you feeling, and how good or bad did you feel?" [34].

During BR, DR, and AR, participants were instructed to record estimated quantities of all food and beverages consumed for ten days during each period of physical testing. The amount and type of food and fluid consumed were calculated using the software program Bilnut (Nutrisoft Bilnut: Food Survey Program version 2.01) and the food-composition tables of the Tunisian National Institute of Statistics (1978) by an expert nutritionist after interviewing all participants.

Furthermore, participants were allowed to bring their own food and drink and consume ad libitum.

Sleep duration, sleep quality, sleep latency, sleep efficiency, sleep disturbances, the use of sleeping medications, and daytime dysfunction were estimated using the Pittsburgh Sleep Quality Index (PSQI), containing 19 questions [35]. The total score of the PSQI ranged from " 0 " (no difficulty)" to "21" (severe difficulties).

The collection of the digit cancelation test, POMS, and PSQI data took place in the laboratory, while the 5mSRT, RPE, and FS data collection took place on a sport field.

During the study, participants fasted for $~ 15-16 \mathrm{~h}$ per day. During BR, DR, and AR, the temperature and relative humidity were respectively $28{ }^{\circ} \mathrm{C}$ and $50 \%, 32{ }^{\circ} \mathrm{C}$ and $49 \%$, and $31^{\circ} \mathrm{C}$ and $47 \%$.

\subsection{The 5-m Shuttle Run Test}

As described by Boukhris et al. [36], participants were required to cover the maximal distance during shuttle sprints of $5 \mathrm{~m}, 10 \mathrm{~m}, 15 \mathrm{~m}, 20 \mathrm{~m}$, etc, until the end of $30 \mathrm{~s}$. This task was performed three times with $35 \mathrm{~s}$ of recovery in between.

During the test, the following parameters were calculated:

- $\quad$ Total distance $(\mathrm{TD})=$ Sum of distances in meter covered during the $6 \times 30$-s shuttles

- $\quad$ Best distance $(\mathrm{BD})=$ The higher distance in meter realized during the $6 \times 30$-s shuttles

- Fatigue index (FI):

$$
\text { FI }(\%)=((\text { shuttle } 1+\text { shuttle } 2)-(\text { shuttle } 5+\text { shuttle } 6)) /(\text { shuttle } 1+\text { shuttle } 2)) \times 100
$$

\subsection{Statistical Analyse}

Data were presented as mean \pm standard deviation (SD) and analyzed using STATISTICA 12.0 Software (Stat-Soft, Maisons-Alfort, Paris, France). Normality of the distribution was confirmed using Shapiro Wilk's test. TD, PSQI, carbohydrates, and lipids data were normally distributed. A two-factor ANOVA (period $\times$ nap) was performed for TD, and a one-factor ANOVA (period) was performed for PSQI, carbohydrates, and lipids data. Post hoc comparisons were completed using the Bonferroni test; effect sizes were calculated as partial eta-squared $\left(\eta_{\mathrm{p}}{ }^{2}\right)$. However, when the normality was not confirmed, a Friedman test was performed [37] for BD, FI, attention, FS, RPE, calorie and protein intake, Hooper indices, and POMS parameters; effect sizes were estimated by Kendall's coefficient of concordance [38]. When significant, pairwise comparisons were also conducted using a Wilcoxon test. Significance was accepted for all analyses at the level of $p \leq 0.05$. Due to the high number of dependent variables, only significant differences are reported.

\section{Results}

\subsection{The 5-m Shuttle Run Test}

\subsubsection{Total Distance}

The repeated measures ANOVA revealed a significant main effect of nap ( $\mathrm{F}=19.29 ; p<0.001$; $\eta_{\mathrm{p}}{ }^{2}=0.59$ ) (Figure 1). The Bonferroni post hoc test revealed a significant increase of TD after N35 
compared with N0 at BR $(\Delta=+4.8 \pm 1.6 \% ; p<0.01)$, DR $(\Delta=+5.1 \pm 1.6 \% ; p<0.01)$, and AR $(\Delta=+5.2 \pm 1.2 \% ; p<0.001)$.

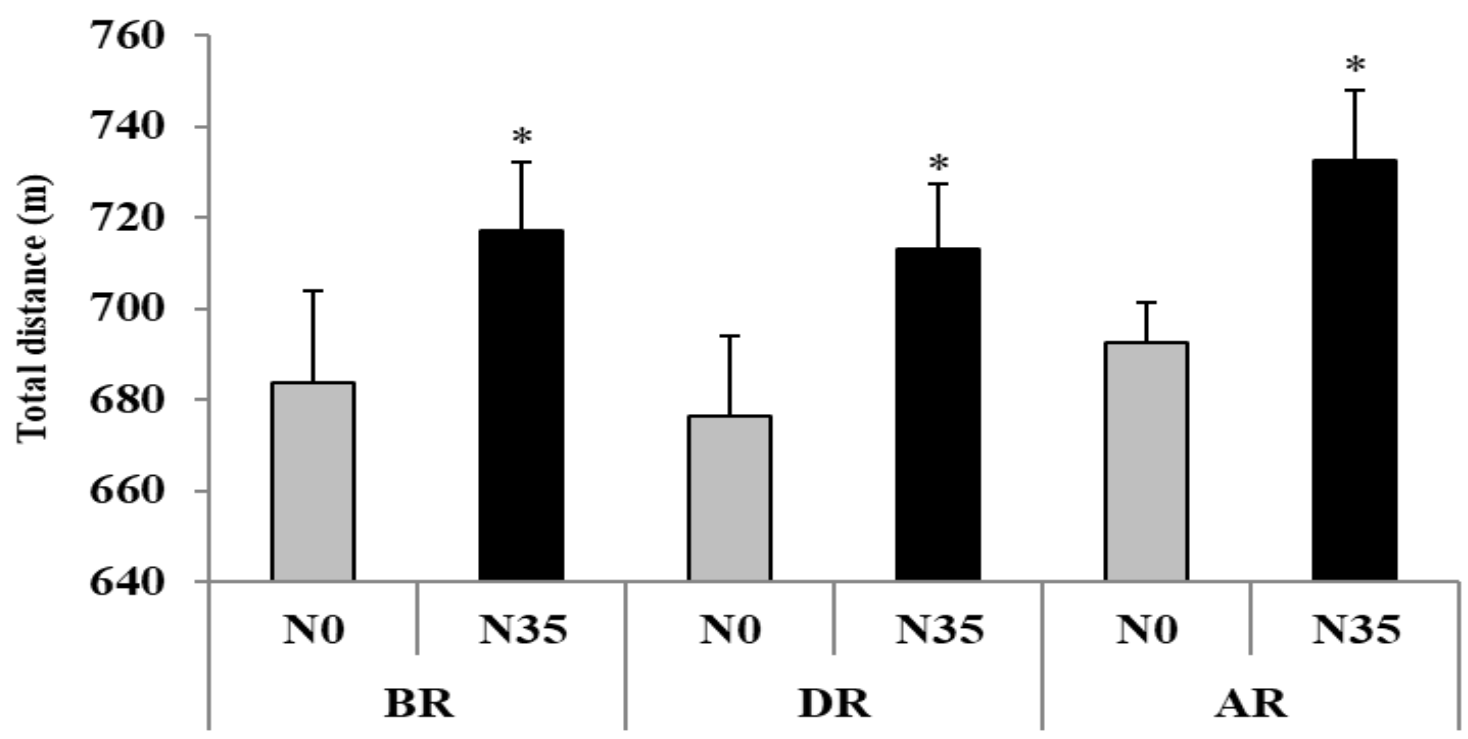

Figure 1. Total distance (mean $\pm \mathrm{SD}$ ) during the 5-m shuttle run test recorded before (BR), during (DR), and after (AR) Ramadan after no-nap (N0) and 35-min nap (N35) opportunities. * Significant difference in comparison with N0.

\subsubsection{Best Distance}

The Friedman test revealed a significant main effect (test $=22.76, p<0.001$, Kendall's W $=0.32$ ) (Figure 2). Pairwise comparisons demonstrated a significant increase of BD after N35 when compared with N0 at BR $(\Delta=+4.4 \pm 2.1 \% ; p<0.05)$, DR $(\Delta=+7.1 \pm 2.2 \% ; p<0.05)$, and $\mathrm{AR}(\Delta=+5.5 \pm 1.5 \%$; $p<0.01)$. However, BD was lower at DR in comparison with AR in N0 $(\Delta=+4.3 \pm 1.3 \% ; p<0.01)$ and $\mathrm{N} 35(\Delta=+2.6 \pm 1.0 \% ; p<0.05)$.

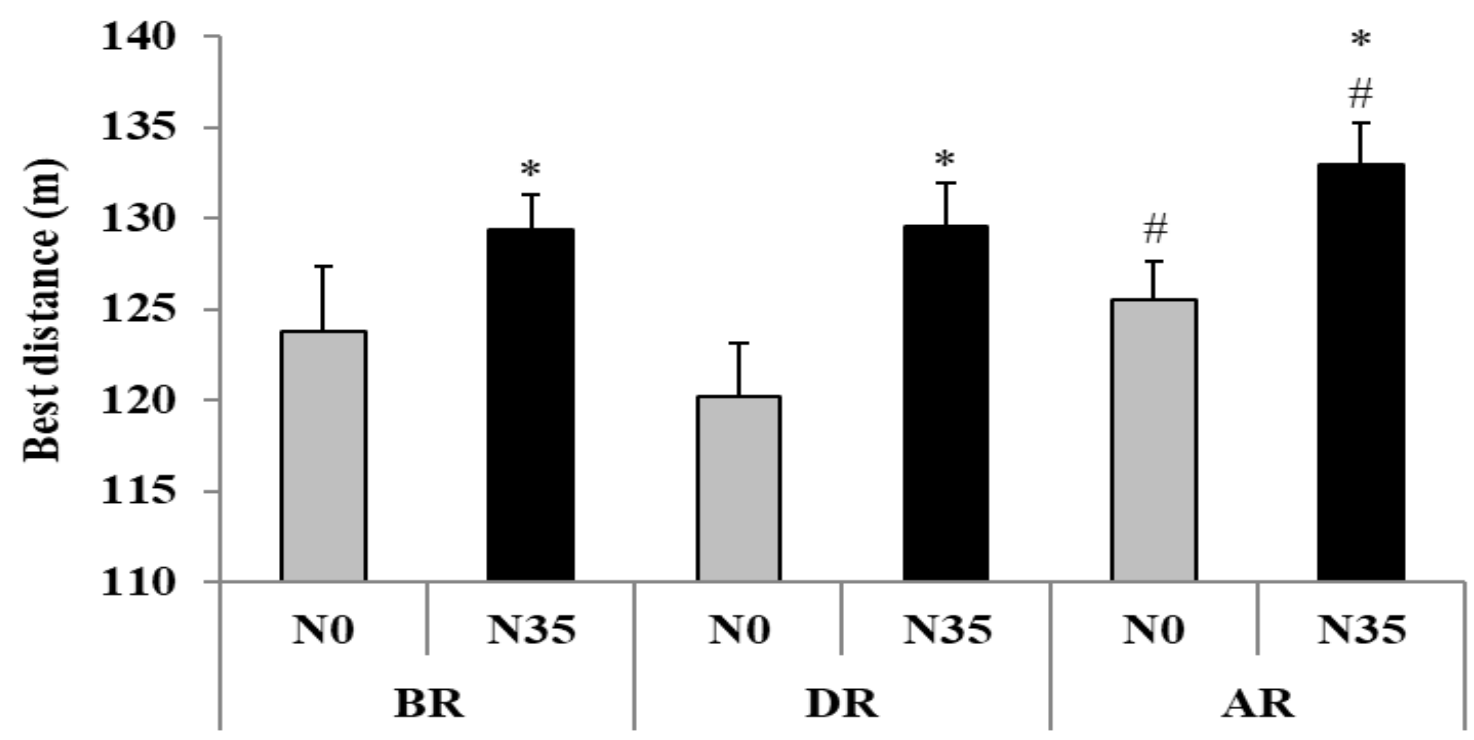

Figure 2. Best distance (mean $\pm \mathrm{SD}$ ) during the 5-m shuttle run test recorded before (BR), during (DR), and after (AR) Ramadan after no-nap (N0) and 35-min nap (N35) opportunities. * Significant difference in comparison with N0; \# significant difference in comparison with DR. 


\subsubsection{Fatigue Index}

The Friedman test did not show any significant main effect (test $=9.72, p>0.05$, Kendall's $\mathrm{W}=0.13$ ) (Figure 3).

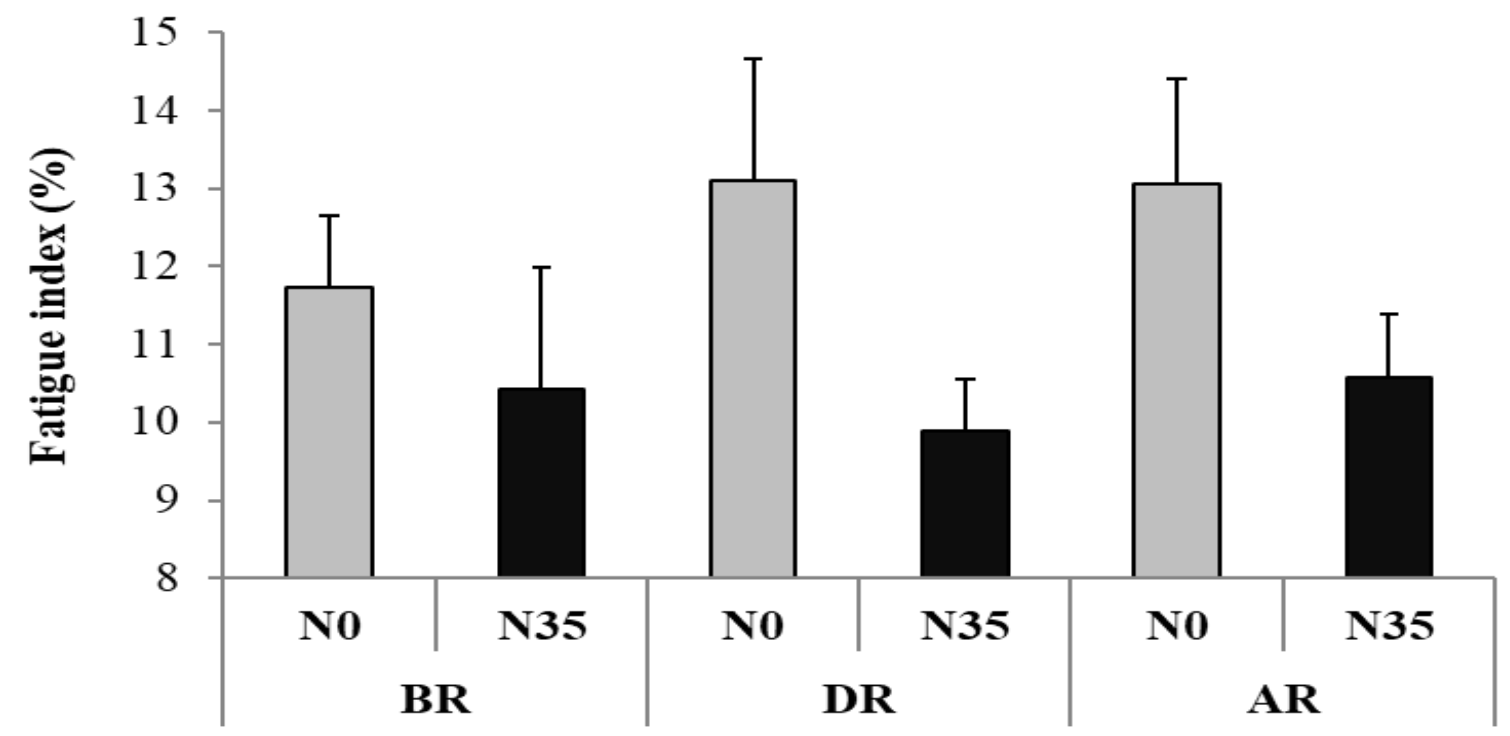

Figure 3. The fatigue index (mean $\pm \mathrm{SD}$ ) during the 5 - $\mathrm{m}$ shuttle run test recorded before (BR), during (DR), and after (AR) Ramadan after no-nap (N0) and 35-min nap (N35) opportunities.

\subsection{Attention Scores Recorded by the Digit Cancellation Test}

Attention scores are presented in Table 1.

Table 1. Feelings, rating of perceived exertion (RPE), and attention scores (mean $\pm \mathrm{SD}$ ) recorded before, during, and after Ramadan after no-nap (N0) and 35-min nap (N35) opportunities.

\begin{tabular}{ccccccc}
\hline \multirow{2}{*}{ Parameters } & \multicolumn{2}{c}{ Before Ramadan } & \multicolumn{2}{c}{ During Ramadan } & \multicolumn{2}{c}{ After Ramadan } \\
\cline { 2 - 6 } & N0 & N35 & N0 & N35 & N0 & N35 \\
\hline $\begin{array}{c}\text { Feelings } \\
\text { Score (a.u) }\end{array}$ & $0.71 \pm 0.36$ & $1.21 \pm 0.37$ & $-0.21 \pm 0.23^{\circ}$ & $0.07 \pm 0.45^{\mathrm{a}, \#}$ & $0.29 \pm 0.24$ & $1.07 \pm 0.21^{*}$ \\
\hline $\begin{array}{c}\text { RPE Score } \\
\text { (a.u) }\end{array}$ & $4.59 \pm 0.28^{\#}$ & $4.22 \pm 0.18$ & $4.32 \pm 0.31^{\#}$ & $3.81 \pm 0.22^{*}$ & $3.92 \pm 0.30$ & $3.73 \pm 0.19$ \\
\hline $\begin{array}{c}\text { Attention } \\
\text { Score (a.u) }\end{array}$ & $64.50 \pm 2.52$ & $67.64 \pm 2.78$ & $61.14 \pm 3.06^{\mathrm{a}, \#}$ & $67.07 \pm 2.86^{*}$ & $65.86 \pm 2.73$ & $75.29 \pm 3.17^{\mathrm{a}, *}$ \\
\hline
\end{tabular}

* Significant difference in comparison with N0; a significant difference in comparison with before Ramadan; and \# significant difference in comparison with after Ramadan.

The Friedman test revealed a significant main effect (test $=35.49, p<0.001$, Kendall's W $=0.5$ ). The Wilcoxon post hoc test showed that attention scores were significantly lower after N0 at DR in comparison with BR $(p<0.05)$ and AR $(p<0.01)$. After N35, attention scores were higher at AR in comparison with BR $(p<0.01)$. However, pairwise comparisons revealed a significant increase in attention after N35 in comparison with N0 at DR $(p<0.01)$ and AR $(p<0.01)$ (Table 1$)$.

\subsection{Rating of Perceived Exertion Scores}

RPE scores are presented in Table 1. The Friedman test revealed a significant main effect (test $=22.84, p<0.001$, Kendall's W $=0.32$ ). Pairwise comparisons revealed a significant decrease of RPE scores recorded after N0 at AR when compared with BR $(p<0.01)$ and DR $(p<0.01)$. Also, RPE scores were significantly lower after N35 in comparison with N0 at DR $(p<0.05)$ (Table 1). 


\subsection{Feelings Scale}

Feelings scores are presented in Table 1 . The Friedman test revealed a significant main effect (test $=19.68, p<0.01$, Kendall's $W=0.28$ ). The Wilcoxon post hoc test showed that feelings scores significantly decreased at DR in comparison with BR after N0 $(p<0.05)$ and after N35 $(p<0.05)$. After N35, feelings scores were significantly higher at AR than DR $(p<0.05)$. Feelings scores were significantly higher at AR after N35 in comparison with N0 $(p<0.05)$ (Table 1).

\subsection{Dietary Intake}

No significant effect of periods on calories intake (test $=1.41 ; p>0.05$; Kendall's $\mathrm{W}=0.05$ ), proteins (test $=0.12, p>0.05$, Kendall's $\mathrm{W}=0.004)$ and lipids $\left(\mathrm{F}=2.4 ; \mathrm{P}>0.05 ; \eta_{\mathrm{p}}{ }^{2}=0.15\right.$ ) was reported. However, statistical analyses revealed a significant effect for carbohydrates $(\mathrm{F}=5.02 ; p<0.05$; $\eta_{\mathrm{p}}{ }^{2}=0.27$ ) (Table 2), wherein carbohydrates were significantly higher during AR in comparison with DR.

Table 2. Dietary intake parameters (mean \pm SD) recorded before, during, and after Ramadan.

\begin{tabular}{cccc}
\hline Parameters & Before Ramadan & During Ramadan & After Ramadan \\
\hline Energy Intake (Kcal/J) & $2696 \pm 142$ & $2439 \pm 113$ & $2410 \pm 145$ \\
\hline Carbohydrates (\%) & $50.5 \pm 1.80$ & $45.86 \pm 2.03$ & $52.51 \pm 1.15^{*}$ \\
\hline Lipids (\%) & $35.07 \pm 2.43$ & $40 \pm 2.34$ & $34.71 \pm 1.27$ \\
\hline Proteins (\%) & $14 \pm 2.13$ & $12.64 \pm 0.59$ & $13 \pm 0.63$ \\
\hline \multicolumn{2}{r}{ * Significant difference in comparison with during Ramadan. }
\end{tabular}

\subsection{The PSQI Questionnaire}

PSQI parameters are presented in Table 3.

Table 3. Pittsburgh Sleep Quality Index (PSQI) parameters (mean \pm SD) recorded before, during, and after Ramadan.

\begin{tabular}{cccc}
\hline Parameters & Before Ramadan & During Ramadan & After Ramadan \\
\hline Sleep Quality (a.u) & $0.86 \pm 0.23$ & $1.93 \pm 0.20^{*}$ & $1.57 \pm 0.23^{*}$ \\
\hline Sleep Latency (min) & $15.36 \pm 1.92$ & $17.57 \pm 2.38$ & $17.43 \pm 1.70$ \\
\hline Sleep Duration (h) & $8 \pm 0.48$ & $6.79 \pm 0.45^{*}$ & $7.21 \pm 0.42$ \\
\hline Sleep Efficiency (\%) & $95.18 \pm 2.36$ & $93.65 \pm 2.09$ & $95.24 \pm 1.83$ \\
\hline Sleep Disturbances (a.u) & $0.57 \pm 0.14$ & $0.79 \pm 0.15$ & $0.64 \pm 0.13$ \\
\hline Daytime Dysfunction (a.u) & $0.21 \pm 0.11$ & $0.79 \pm 0.21^{*}$ & $0.29 \pm 0.13$ \\
\hline Total PSQI Scores (a.u) & $3.07 \pm 0.65$ & $6.07 \pm 0.68^{*}$ & $4.64 \pm 0.56 *$ *\# \\
\hline
\end{tabular}

* Significant difference in comparison with before Ramadan; \# significant difference in comparison with during Ramadan.

No significant effect was reported for sleep latency (test $=2.07, p>0.05$, Kendall's $W=0.07$ ), sleep efficiency (test $=3.5, p>0.05$, Kendall's $W=0.12$ ), or sleep disturbances (test $=4.66, p>0.05$, Kendall's $\mathrm{W}=0.16)$ (Table 3$)$.

\subsubsection{Sleep Quality}

A significant effect was reported (test $=14.97, p<0.0005$, Kendall's $\mathrm{W}=0.53$ ). The sleep quality scores increased significantly at DR $(p<0.01)$ and AR $(p<0.05)$ in comparison with BR. 


\subsubsection{Sleep Duration}

There was a significant effect (test $=8.21, p<0.05$, Kendall's $\mathrm{W}=0.29$ ) with decrease in sleep duration DR in comparison with BR $(p<0.05)$.

\subsubsection{Daytime Dysfunction}

There was a significant effect (test $=8.43, p<0.05$, Kendall's $W=0.3$ ) with higher sleep dysfunction scores DR in comparison with BR $(p<0.05)$.

\subsubsection{Total PSQI Scores}

The statistical analysis revealed a significant effect $\left(\mathrm{F}=18.59 ; p<0.001 ; \eta_{\mathrm{p}}{ }^{2}=0.59\right)$. Total PSQI scores were significantly higher in DR $(p<0.001)$ and AR $(p<0.05)$ in comparison with BR.

\subsection{Hooper Questionnaire Indices}

Sleep, stress, fatigue, and muscle soreness scores are presented in Table 4.

Table 4. Sleep, stress, fatigue, and muscle soreness scores (mean $\pm \mathrm{SD}$ ) recorded before, during, and after Ramadan after no-nap (N0) and 35-min nap (N35) opportunities.

\begin{tabular}{ccccccc}
\hline \multirow{2}{*}{ Parameters } & \multicolumn{2}{c}{ Before Ramadan } & \multicolumn{2}{c}{ During Ramadan } & \multicolumn{2}{c}{ After Ramadan } \\
\cline { 2 - 6 } & N0 & N35 & N0 & N35 & N0 & N35 \\
\hline Sleep (a.u) & $3.86 \pm 0.47$ & $3.43 \pm 0.31$ & $4.50 \pm 0.45$ & $4.07 \pm 0.40$ & $4.43 \pm 0.31$ & $4.00 \pm 0.21$ \\
\hline Stress (a.u) & $3.43 \pm 0.33$ & $3.07 \pm 0.38$ & $2.50 \pm 0.37$ & $3.00 \pm 0.42$ & $2.71 \pm 0.37$ & $2.36 \pm 0.31$ \\
\hline Fatigue (a.u) & $3.93 \pm 0.30$ & $4.00 \pm 0.30$ & $3.93 \pm 0.47$ & $3.79 \pm 0.37$ & $4.57 \pm 0.27^{*}$ & $3.64 \pm 0.13^{\#}$ \\
\hline Muscle Soreness (a.u) & $3.36 \pm 0.40$ & $3.14 \pm 0.33$ & $2.93 \pm 0.34$ & $2.57 \pm 0.25$ & $3.21 \pm 0.26$ & $2.86 \pm 0.29$ \\
\hline
\end{tabular}

* Significant difference in comparison with before Ramadan; \# significant difference with N0.

No significant period was reported for sleep (test $=7.96, p>0.05$, Kendall's $W=0.11$ ), stress (test $=10.23, p>0.05$, Kendall's $\mathrm{W}=0.14$ ), or muscle soreness (test $=7.68, p>0.05$, Kendall's $\mathrm{W}=0.10$ ) (Table 4).

For the fatigue scores, the Friedman test revealed a significant main effect (test $=11.74, p<0.05$, Kendall's $W=0.16$ ). Pairwise comparisons revealed significant increases in fatigue scores for AR compared with BR $(p<0.05)$ after N0. At AR, the fatigue scores were significantly lower after N35 compared to N0 $(p<0.01)$.

\subsection{The Profile of Mood State}

The Friedman test did not show any significant effect on total mood scores (test $=3.34, p>0.05$, Kendall's $\mathrm{W}=0.04$ ), tension (test $=3.78, p>0.05$, Kendall's $\mathrm{W}=0.05$ ), anger (test $=4.69, p>0.05$, Kendall's $W=0.06$ ), confusion (test $=0.77, p>0.05$, Kendall's $W=0.01$ ), depression (test $=7.74$, $p>0.05$, Kendall's $W=0.11$ ), fatigue (test $=9.62, p>0.05$, Kendall's $W=0.13$ ), and vigor (test $=9.04$, $p>0.05$, Kendall's W $=0.12$ ) (Table 5). 
Table 5. Profile of mood state (POMS) parameters (mean $\pm \mathrm{SD}$ ) recorded before (BR), during (DR), and after (AR) Ramadan after no-nap (N0) and 35-min nap (N35) opportunities.

\begin{tabular}{ccccccc}
\hline \multirow{2}{*}{ Parameters } & \multicolumn{2}{c}{ Before Ramadan } & \multicolumn{2}{c}{ During Ramadan } & \multicolumn{2}{c}{ After Ramadan } \\
\cline { 2 - 7 } & N0 & N35 & N0 & N35 & N0 & N35 \\
\hline Total Mood Scores (a.u) & $23.21 \pm 9.02$ & $16.50 \pm 8.45$ & $21.21 \pm 9.36$ & $17.07 \pm 8.82$ & $24.21 \pm 7.99$ & $15.50 \pm 4.93$ \\
\hline Tension (a.u) & $9.57 \pm 1.76$ & $8.86 \pm 1.56$ & $9.57 \pm 1.66$ & $7.86 \pm 1.62$ & $9.14 \pm 1.37$ & $7.93 \pm 1.21$ \\
\hline Anger (a.u) & $11.37 \pm 2.58$ & $9.07 \pm 1.91$ & $9.43 \pm 2.59$ & $8.57 \pm 2.38$ & $9.64 \pm 1.95$ & $7.93 \pm 1.62$ \\
\hline Confusion (a.u) & $6.86 \pm 1.14$ & $6.36 \pm 1.45$ & $6.57 \pm 1.13$ & $6.21 \pm 1.13$ & $7 \pm 1.11$ & $6.79 \pm 1.05$ \\
\hline Depression (a.u) & $8.07 \pm 2.42$ & $7.64 \pm 2.39$ & $6.79 \pm 2.73$ & $6.79 \pm 2.94$ & $9 \pm 2.48$ & $7.71 \pm 1.70$ \\
\hline Fatigue (a.u) & $6.71 \pm 1.56$ & $4.93 \pm 1.30$ & $6 \pm 1.62$ & $4.71 \pm 1.47$ & $6.43 \pm 1.47$ & $3.79 \pm 0.85$ \\
\hline Vigor (a.u) & $19.36 \pm 1.78$ & $20.36 \pm 1.64$ & $17.14 \pm 2.04$ & $17.07 \pm 1.64$ & $17 \pm 1.82$ & $16.07 \pm 2.07$ \\
\hline
\end{tabular}

\section{Discussion}

The purpose of this study was to investigate the effects of a 35-min nap on physical performance during the 5mSRT; attention; feelings; mood states; and the perception of stress, muscle soreness, and fatigue before, during and after Ramadan observance in young physically active men. The main findings of the present study were as follows: (i) TD and BD significantly improved after N35 in comparison with N0 at BR, DR, and AR and (ii) attention was significantly higher after N35 in comparison with $\mathrm{N} 0$ at $\mathrm{DR}$ and AR.

During N0, findings revealed that TD and FI were not affected by Ramadan observance; this supports the results of Boukhris et al. [36], who reported that TD and FI during the 5mSRT were not significantly affected by the fasting period. As previously suggested by Boukhris et al. [36], the maintained TD during Ramadan observance could be related to the unchanged total fat intake during Ramadan. In fact, fat oxidation could assist endurance performance by mitigating the onset of fatigue via the sparing of muscle glycogen [39]. Additionally, Chtourou et al. [12] suggested that perturbation in mood states is one of the main reasons for impeded performance during Ramadan. Supporting our findings, mood state parameters, stress, sleep, and muscle soreness were unaffected by Ramadan, which may have assisted the unchanged TD and FI.

However, in contradiction with the results of Boukhris et al. [36], this study demonstrated that BD was significantly lower in DR compared to AR $(120.87 \mathrm{~m} \pm 2.89 \mathrm{~m}$ vs. $125.8 \mathrm{~m} \pm 2.1 \mathrm{~m})$. This result is in agreement with those of Chtourou et al. [12], who reported that performances during repeated cycling exercise and the Wingate test were affected by Ramadan fasting in young footballers. Chtourou et al. [12] explained this impairment by the fact that fatigue was higher in DR compared to BR. Similarly, RPE scores recorded after the 5mSRT were significantly increased in DR in comparison with AR. Also, feelings scores recorded after the $5 \mathrm{mSRT}$ were lower in DR than BR. Thus, the increased perception of fatigue and the reduced feelings state could explain high performance attainment in DR. This contradiction between the present results and those of Boukhris et al. [36] could be explained by the fact that sleep scores in the present study were negatively affected by Ramadan observance. Thus, worse sleep impacted the ability to perform at the highest intensity during the 5mSRT. Also, BD (i.e., registered in all cases for the first 30-s repetition) is possibly related to alactic energy usage or adenosine triphosphate and phosphocreatine (ATP-PCr) capacity. The decreased fractional contribution of carbohydrate may also explain the decline of BD during DR.

Attention estimated by the digit cancellation test was significantly lower at DR in comparison with BR and AR; this supports the results of Boukhris et al. [11], who reported that Ramadan observance had an adverse effect on attention. Also, supporting previous literature $[4,5,11]$, the impaired sleep reported in DR could be related to a decrement in cognitive performance, such as an attention task. Indeed, there was a significant increase in total scores on the PSQI and the subjective sleep quality scores DR in comparison with BR, with a significant decrease of sleep duration in DR compared to BR. In addition, although total 
PSQI scores were higher at AR in comparison with BR, it does not indicate poor sleep quality. In fact, PSQI scores reported during Ramadan (PSQI scores $=4.64$ ) were above 5 [40].

It was hypothesized that a 35-min nap may be beneficial for all measured parameters both outside and during Ramadan. This hypothesis was partially confirmed. Although no changes have been reported for the perception of mood states, stress, sleep, muscle soreness, and the FI during the 5mSRT, BD and HD were better after N35 compared to N0 at BR, DR, and AR. Additionally, the other parameters reached significance (N35 better than N0) only in some periods of study: attention at DR and AR, RPE at DR, feelings at AR, and fatigue estimation at AR. In contradiction, Hsouna et al. [29] did not report a significant effect of the 25-min nap opportunity on physical performance during the 5mSRT and attention. However, in support of the speculation of the study of Hsouna et al. [28], increasing the nap opportunity to $35 \mathrm{~min}$ allowed for significant positive effects on cognitive and short-term repetitive maximal performances. The benefits observed only after a 35-min nap and not after a 25-min nap could be related to increased inclusion of slow-wave sleep, known as a recovery period for daily metabolism [26]. Previous studies, out of Ramadan, have reported that a 35-min nap opportunity is beneficial for performance improvement and perception of fatigue reduction during the 5mSRT [26]. To the best of the authors' knowledge, this is the first study investigating the effect of a nap opportunity during Ramadan observance on physical and cognitive performance. Although positive effects have been observed on physical performance (i.e., increase of TD and BD after N35 in the all experimental sessions) and cognitive performance (i.e., improvement of attention after N35 at DR and AR), previous studies show that napping might improve physical $[24-28,41]$ and cognitive $[28,41]$ performance. The non significant effect of the nap opportunity on some parameters may be related to duration. It is possible that, if the nap time was increased, significant differences may have been observed for a majority of the dependent variables. For example, with lower duration, Petit et al. [42] reported that a 20-min nap did not improve performance during the 30-s Wingate test after normal or 5-h phase advanced sleep. In this context, Hammouda et al. [25] concluded that better improvement in physical performance was recorded after the longer nap duration (i.e., after a 90-min compared to 20-min nap opportunity). Boukhris et al. [26] reported that, compared to $25 \mathrm{~min}$ and $35 \mathrm{~min}, 45 \mathrm{~min}$ is a better duration for improving the $5 \mathrm{mSRT}$ performance. Additionally, Hsouna et al. [28] concluded that the beneficial effect of a nap after a normal sleep night on the 5-jump test performance exists when the nap duration was over $35 \mathrm{~min}$. It has been reported that the improvement of physical performance is associated with a reduction of subjective fatigue [26,43], feelings scores [28], and an improvement in alertness [43]. These findings are, partially, in agreement with the results of the present study, which confirmed a decrease of RPE scores after N35 at DR, an improvement of attention, and the estimated fatigue at AR.

Although the present study presented important practical recommendations, some limitations exist. First, the present study lacks an objective measurement of the night and the nap sleep (e.g., by the use of actigraphy or polysomnography). Future studies should utilize registration of the previous night's sleep and the nap during the experimental period. In addition, a specific sleep scale relating to quality of sleep during the nap should be used for future studies. Moreover, due to a technical issue, the current study lacks control of the luminosity level during the sleeping period; this drawback should be avoided in future studies. In addition, to confirm the results of the present study, further studies should compare different nap durations outside and during Ramadan.

\section{Conclusions}

The results revealed that TD and FI during the 5mSRT were not affected by Ramadan observance. However, BD was significantly lower in DR compared to AR after N0 and N35. During N0 condition, attention decreased significantly in DR in comparison with BR and AR. BD and TD were better after N35 compared to N0 at BR, DR, and AR. A significant increase of attention after N35 in comparison with N0 at DR and AR was observed. However, no changes have been reported for the perception of mood states, stress, sleep, muscle soreness, and FI during the 5mSRT. Additionally, N35 was better than 
N0 for attention at DR and AR, RPE at DR, feelings at AR, and fatigue estimation at AR. We suggested that further increases of the nap duration during Ramadan would allow for significant effects of the nap in all parameters before, during, and after the fasting month.

Therefore, coaches and athletes should plan some periods of 35-min daytime naps during Ramadan observance to overcome any sleep loss and/or to improve physical and cognitive performances.

Author Contributions: Conceptualization, K.T., N.L.B., and H.C.; methodology, H.H., O.B., K.T., N.L.B., and H.C.; software, H.H.; validation, H.H.; formal analysis, H.H.; investigation, H.H.; resources, N.L.B.; data curation, H.H., O.B., K.T., and H.C.; writing-original draft preparation, H.H., O.B., K.T., and H.C.; writing-review and editing, H.H., O.B., K.T., R.A., A.A., J.M.G., N.B., N.S., P.L., S.G., N.L.B., and H.C.; visualization, H.H.; supervision, K.T., N.L.B., and H.C.; project administration, H.C.; funding acquisition, N.L.B. All authors have read and agreed to the published version of the manuscript.

Funding: This research received no external funding.

Conflicts of Interest: The authors declare no conflict of interest.

\section{References}

1. Chtourou, H. Effects of Ramadan Fasting on Health and Athletic Performance; Omics Group International: New York, NY, USA, 2015; pp. 6-14.

2. Chtourou, H.; Chtourou, L.; Trabelsi, K.; Tahri, N.; Souissi, N. Possible gastrointestinal disorders for athletes during Ramadan: An overview. Biol. Rhythm Res. 2018, 49, 51-60. [CrossRef]

3. Chtourou, H.; Hammouda, O.; Aloui, A.; Souissi, N.; Chaouachi, A. The optimal time of day for training during Ramadan: A review study. J. Fast. Health 2014, 2, 46-52.

4. Trabelsi, K.; Stannard, S.R.; Chtourou, H.; Moalla, W.; Ghozzi, H.; Jamoussi, K.; Hakim, A. Monitoring athletes' hydration status and sleep patterns during Ramadan observance: Methodological and practical considerations. Biol. Rhythm Res. 2018, 49, 337-365. [CrossRef]

5. Trabelsi, K.; Bragazzi, N.; Zlitni, S.; Khacharem, A.; Boukhris, O.; El-Abed, K.; Ammar, A.; Khanfir, S.; Shephard, R.J.; Hakim, A.; et al. Observing Ramadan and sleep-wake patterns in athletes: A systematic review, meta-analysis and meta-regression. Br. J. Sports Med. 2020, 54, 674-680. [CrossRef]

6. Trabelsi, K.; Shephard, R.J.; Boukhris, O.; Ammar, A.; Khanfir, S.; Hakim, A.; Bragazzi, N.L.; Chtourou, H. Effects of Ramadan fasting on hematological indices in athletes: A systematic review. Tunis Med. 2019, 97, 1104-1113.

7. Trabelsi, K.; Chtourou, H. Teaching physical education during Ramadan observance: Practical recommendations. Int. J. Sport Stud. Health 2019, 2019. [CrossRef]

8. Boukhris, O.; Trabelsi, K.; Chtourou, H. Evolution of dietary intake between before, during and after Ramadan observance in Tunisian physically active men: A Systematic Review. Int. J. Sport. Stud. Health 2018, 1, e83782. [CrossRef]

9. Aloui, A.; Baklouti, H.; Souissi, N.; Chtourou, H. Effects of Ramadan fasting on body composition in athletes: A systematic review Effets du jeûne du Ramadan sur la composition corporelle des sportifs: Revue systématique. Tunis Med. 2019, 97, 1087-1094.

10. Chaouachi, A.; Coutts, A.J.; Chamari, K.; Wong, D.P.; Chaouachi, M.; Chtara, M.; Amri, M. Effect of Ramadan intermittent fasting on aerobic and anaerobic performance and perception of fatigue in male elite judo athletes. J. Strength Cond Res. 2009, 23, 2702-2709. [CrossRef]

11. Boukhris, O.; Trabelsi, K.; Shephard, R.J.; Hsouna, H.; Abdessalem, R.; Chtourou, L.; Ammar, A.; Bragazzi, N.L.; Chtourou, H. Sleep Patterns, Alertness, Dietary Intake, Muscle Soreness, Fatigue, and Mental Stress Recorded before, during and after Ramadan Observance. Sports 2019, 7, 118. [CrossRef]

12. Chtourou, H.; Hammouda, O.; Souissi, H.; Chamari, K.; Chaouachi, A.; Souissi, N. The effect of Ramadan fasting on physical performances, mood state and perceived exertion in young footballers. Asian J. Sports Med. 2011, 2, 177. [CrossRef] [PubMed]

13. Chtourou, H.; Hammouda, O.; Chaouachi, A.; Chamari, K.; Souissi, N. The effect of time of-day and Ramadan fasting on anaerobic performances. Int. J. Sports Med. 2012, 33, 142-147. [CrossRef] [PubMed] 
14. Hsouna, H.; Abdessalem, R.; Boukhris, O.; Trabelsi, K.; Chtourou, L.; Tahri, N.; Engel, F.A.; Shephard, R.J.; Chtourou, H. Short-term maximal performance, alertness, dietary intake, sleep pattern and mood states of physically active young men before, during and after Ramadan observance. PLoS ONE. 2019, 14, e0217851. [CrossRef] [PubMed]

15. Chtourou, H.; Trabelsi, K.; Boukhris, O.; Ammar, A.; Shephard, R.J.; Bragazzi, N.L. Effects of Ramadan fasting on physical performances in soccer players: A systematic review effets du jeûne de Ramadan sur les performances physiques des footballeurs: Revue systématique. Tun Med. 2019, 97, 1114-1131.

16. Baklouti, H.; Rejeb, N.; Aloui, A.; Jaafar, H.; Ammar, A.; Chtourou, H.; Girard, O.; Souissi, N. Short versus long small-sided game training during Ramadan in soccer players. Phys. Ther. Sport 2017, 24, 20-25. [CrossRef]

17. Chamari, K.; Briki, W.; Farooq, A.; Patrick, T.; Belfekih, T.; Herrera, C.P. Impact of Ramadan intermittent fasting on cognitive function in trained cyclists: A pilot study. Biol. Sport 2016, 33, 49. [CrossRef]

18. Zerguini, Y.; Kirkendall, D.; Junge, A.; Dvorak, J. Impact of Ramadan on physical performance in professional soccer players. Br. J. Sports Med 2007, 41, 398-400. [CrossRef]

19. Jarraya, M.; Jarraya, S.; Chtourou, H.; Souissi, N.; Chamari, K. The effect of partial sleep deprivation on the reaction time and the attentional capacities of the handball goalkeeper. Biol. Rhythm Res. 2013, 44, 503-510. [CrossRef]

20. Jarraya, S.; Jarraya, M.; Chtourou, H.; Souissi, N. Effect of time of day and partial sleep deprivation on the reaction time and the attentional capacities of the handball goalkeeper. Biol. Rhythm Res. 2014, 45, 183-191. [CrossRef]

21. Souissi, N.; Chtourou, H.; Aloui, A.; Hammouda, O.; Dogui, M.; Chaouachi, A.; Chamari, K. Effects of time-of-day and partial sleep deprivation on short-term maximal performances of judo competitors. J. Strength Cond Res. 2013, 27, 2473-2480. [CrossRef]

22. HajSalem, M.; Chtourou, H.; Aloui, A.; Hammouda, O.; Souissi, N. Effects of partial sleep deprivation at the end of the night on anaerobic performances in judokas. Biol. Rhythm Res. 2013, 44, 815-821. [CrossRef]

23. Nédélec, M.; Halson, S.; Abaidia, A.E.; Ahmaidi, S.; Dupont, G. Stress, sleep and recovery in elite soccer: A critical review of the literature. Sports Med. 2015, 45, 1387-1400. [CrossRef] [PubMed]

24. Waterhouse, J.; Atkinson, G.; Edwards, B.; Reilly, T. The role of a short post-lunch nap in improving cognitive, motor, and sprint performance in participants with partial sleep deprivation. J. Sports Sci. 2007, 25, 1557-1566. [CrossRef] [PubMed]

25. Hammouda, O.; Romdhani, M.; Chaabouni, Y.; Mahdouani, K.; Driss, T.; Souissi, N. Diurnal napping after partial sleep deprivation affected hematological and biochemical responses during repeated sprint. Biol. Rhythm Res. 2018, 49, 927-939. [CrossRef]

26. Boukhris, O.; Abdessalem, R.; Ammar, A.; Hsouna, H.; Trabelsi, K.; Engel, F.A.; Sperlich, B.; Hill, D.W.; Chtourou, H. Nap opportunity during the daytime affects performance and perceived exertion in 5-m shuttle run test. Front. Physiol. 2019, 10, 779. [CrossRef] [PubMed]

27. Abdessalem, R.; Boukhris, O.; Hsouna, H.; Trabelsi, K.; Ammar, A.; Taheri, M.; Irandoust, K.; Hill, D.W.; Chtourou, H. Effect of napping opportunity at different times of day on vigilance and shuttle run performance. Chronobiol. Int. 2019, 36, 1334-1342. [CrossRef]

28. Hsouna, H.; Boukhris, O.; Abdessalem, R.; Trabelsi, K.; Ammar, A.; Shephard, R.J.; Chtourou, H. Effect of different nap opportunity durations on short-term maximal performance, attention, feelings, muscle soreness, fatigue, stress and sleep. Physiol. Behav. 2019, 211, 112673. [CrossRef]

29. Hsouna, H.; Boukhris, O.; Trabelsi, K.; Abdessalem, R.; Ammar, A.; Irandoust, K.; Taheri, M.; Souissi, N.; Shephard, R.J.; Garbarino, S.; et al. Effects of 25-Min Nap Opportunity during Ramadan Observance on the 5-m Shuttle Run Performance and the Perception of Fatigue in Physically Active Men. Int. J. Environ. Res. Public Health 2020, 17, 3135. [CrossRef]

30. Herrera, C.P. Total sleep time in Muslim football players is reduced during Ramadan: A pilot study on the standardized assessment of subjective sleep-wake patterns in athletes. J. Sports Sci. 2012, 30, S85-S91. [CrossRef]

31. Hooper, S.L.; Mackinnon, L.T.; Howard, A.L.F.; Gordon, R.D.; Bachmann, A.W. Markers for monitoring overtraining and recovery. Med. Sci. Sports Exerc. 1995, 27, 106-112. [CrossRef]

32. Cayrou, S.; Dickès, P.; Dolbeault, S. Version française du profile of mood states (POMS-f). J. Ther. Comport. Cogn. 2003, 13, 83-88. 
33. Haddad, M.; Chaouachi, A.; Castagna, C.; Hue, O.; Wong, D.P.; Tabben, M.; Behm, D.G.; Chamari, K. Validity and psychometric evaluation of the French version of RPE scale in young fit males when monitoring training loads. Sci. Sports 2013, 28, 29-35. [CrossRef]

34. Hardy, C.J.; Rejeski, W.J. Not what, but how one feels: The measurement of affect during exercise. J. Sport Exerc. Psychol. 1989, 11, 304-317. [CrossRef]

35. Suleiman, K.H.; Yates, B.C.; Berger, A.M.; Pozehl, B.; Meza, J. Translating the Pittsburgh sleep quality index into Arabic. Western J. Nurs. Res. 2010, 32, 250-268. [CrossRef] [PubMed]

36. Boukhris, O.; Hsouna, H.; Chtourou, L.; Abdesalem, R.; BenSalem, S.; Tahri, N.; Trabelsi, K.; Stannard, S.R.; Chtourou, H. Effect of Ramadan fasting on feelings, dietary intake, rating of perceived exertion and repeated high intensity short-term maximal performance. Chronobiol. Int. 2019, 36, 1-10. [CrossRef] [PubMed]

37. Sheldon, M.R.; Fillyaw, M.J.; Thompson, W.D. The use and interpretation of the Friedman test in the analysis of ordinal-scale data in repeated measures designs. Physiother. Res. Int. 1996, 1, 221-228. [CrossRef]

38. Field, A.P. K endall's Coefficient of Concordance. Wiley StatsRef: Statistics Reference Online; Encyclopedia of Statistics in Behavioral Science: London, UK, 2014.

39. Horvath, P.J.; Eagen, C.K.; Fisher, N.M.; Leddy, J.J.; Pendergast, D.R. The effects of varying dietary fat on performance and metabolism in trained male and female runners. J. Am. Coll. Nutr. 2000, 19, 52-60. [CrossRef]

40. Buysse, D.J.; Ancoli-Israel, S.; Edinger, J.D.; Lichstein, K.L.; Morin, C.M. Recommendations for a standard research assessment of insomnia. Sleep 2006, 29, 1155-1173. [CrossRef]

41. Daaloul, H.; Souissi, N.; Davenne, D. Effects of Napping on Alertness, Cognitive, and Physical Outcomes of Karate Athletes. Med. Sci. Sports Exerc. 2019, 51, 338-345. [CrossRef]

42. Petit, E.; Mougin, F.; Bourdin, H.; Tio, G.; Haffen, E. A 20-min nap in athletes changes subsequent sleep architecture but does not alter physical performances after normal sleep or 5-h phase-advance conditions. Eur. J. Appl. Physiol. 2014, 114, 305-315. [CrossRef]

43. Brooks, A.; Lack, L. A brief afternoon nap following nocturnal sleep restriction: Which nap duration is most recuperative? Sleep 2006, 29, 831-840. [CrossRef] [PubMed]

(C) 2020 by the authors. Licensee MDPI, Basel, Switzerland. This article is an open access article distributed under the terms and conditions of the Creative Commons Attribution (CC BY) license (http://creativecommons.org/licenses/by/4.0/). 